\title{
Nano-scale encapsulation enhances allograft survival and function of islets transplanted in a mouse model of diabetes
}

\author{
Z.-I. Zhi • A. Kerby • A. J. F. King • P. M. Jones • \\ J. C. Pickup
}

Received: 26 September 2011 / Accepted: 30 November 2011 /Published online: 14 January 2012

(C) Springer-Verlag 2012

\begin{abstract}
Aims/hypothesis The success of islet transplantation as a treatment for type 1 diabetes is currently hampered by post-transplantation loss of functional islets through adverse immune and non-immune reactions. We aimed to test whether early islet loss can be limited and transplant survival improved by the application of conformal nano-coating layers to islets.

Methods Our novel coating protocol used alternate layers of phosphorylcholine-derived polysaccharides (chitosan or chondroitin-4-sulphate) and alginate as coating materials, with the binding based on electrostatic complexation. The in vitro function of encapsulated mouse islets was studied by analysing islet secretory function and cell viability. The in vivo function was evaluated using syngeneic and allogeneic transplantation in the streptozotocin-induced mouse model of diabetes.

Results Nano-scale encapsulated islets retained appropriate islet secretory function in vitro and were less susceptible to complement- and cytokine-induced apoptosis than nonencapsulated control islets. In in vivo experiments using a syngeneic mouse transplantation model, no deleterious responses to the coatings were observed in host animals, and the encapsulated islet grafts were effective in reversing hyperglycaemia. Allo-transplantation of the nano-coated islets resulted in preserved islet function post-implantation
\end{abstract}

Electronic supplementary material The online version of this article (doi:10.1007/s00125-011-2431-y) contains peer-reviewed but unedited supplementary material, which is available to authorised users.

Z.-1. Zhi $(\bowtie) \cdot$ A. Kerby · A. J. F. King • P. M. Jones · J. C. Pickup Diabetes Research Group, King's College London School of Medicine, Guy's Hospital Campus,

London SE1 1UL, UK

e-mail: z.zhi@kcl.ac.uk in five of seven mice throughout the 1 month monitoring period.

Conclusions/interpretation Nano-scale encapsulation offers localised immune protection for implanted islets, and may be able to limit early allograft loss and extend survival of transplanted islets. This versatile coating scheme has the potential to be integrated with tolerance induction mechanisms, thereby achieving long-term success in islet transplantation.

Keywords Allogeneic transplantation · Graft survival · Immunoisolation · Islet transplantation · Nano-coating · Nano-scale encapsulation · Type 1 diabetes
Abbreviations
HBSS Hanks' buffered salt solution
PC Phosphorylcholine
PEG Polyethylene-glycol
TEM Transmission electron microscopy

\section{Introduction}

Islet transplantation is arguably one of the most important conceptual advances in the treatment of type 1 diabetes and has the potential to cure the disease [1]. A major barrier to successful transplantation is early post-transplantation depletion of functional islets in response to activated complement and coagulation systems, and to a chronic inflammatory and immunogenic environment [2-5]. This has obvious detrimental effects on the outcome of individual grafts and further exacerbates the scarcity of donor tissue. Moreover, allogeneic transplantation requires pharmacological suppression of the host immune system to circumvent graft rejection, while current immunosuppressive regimens are likely to contribute 
to the early loss of engrafted islets [6]. A strategy that conceals implanted islets from the host immune system may help to minimise or even prevent the post-transplantation loss of islet mass and function by immune rejection. Thus physical isolation of islets from the host environment by encapsulation, while maintaining islet responsiveness to metabolic changes, could be an effective means of improving islet survival and function after transplantation.

Islet encapsulation strategies to date have mainly focused on macrocapsules (encapsulation of the whole islet graft) and microcapsules (encapsulation of individual islets) [7]. Previous studies in animal models [8,9] and in human participants $[10,11]$ have demonstrated that physical isolation of islets from the host immune system by, for example, alginate microencapsulation is effective in preventing beta cell loss and in maintaining long-term secretory function in transplanted allogeneic and xenogeneic islets without systemic immunosuppression [12-16]. Although conferring the immunological advantages predicted from graft/host isolation, microcapsules have some significant drawbacks. Thus the relatively large volume of a typical alginate capsule (diameter $\sim 500-800 \mu \mathrm{m}$ ) compared with a typical islet $(\sim 150 \mu \mathrm{m})$ results in a greatly increased diffusion length, which can lead to impaired diffusion of oxygen and nutrients to the islet, with consequent hypoxic cell death or malfunction. A more immediate consequence of the size of microcapsules is the choice of anatomical location for the graft material, with most experimental studies focussing on intraperitoneal or subcutaneous compartments due to their large capacity [17]. In contrast, intraportal infusion is the current site of choice for clinical programmes [1], with the transplanted islets lodging in the hepatic microcirculation. An encapsulation technology designed for application to current clinical transplantation of human islets must therefore be compatible with intraportal delivery to the hepatic capillary bed, excluding microcapsules or macrocapsules on the basis of their size.

Conformal nano-coating avoids these problems by generating a biocompatible nanometre-scale isolating layer close to the cell surface, thus reducing barriers to diffusion, while ensuring that the encapsulated islets can be implanted into any site suitable for non-encapsulated islets [7]. The challenge with conformal nano-coating is to nano-engineer an efficient and lasting immune-protective layer that covers islets completely or nearly completely, and is biocompatible with the recipient.

Several methods of conformal coating have been developed recently, including covalent surface attachment of polyethylene glycol (PEG), known as 'PEGylation' [18], and layer-by-layer encapsulation [19-28]. Nano-layers with different combinations of components, including streptavidin and biotin-PEG derivatives [19, 24], complement receptor 1 and heparin [25], and PEG-lipid and poly(vinyl alcohol)
$[26,27]$, have been previously researched as islet surface coatings. Most of these attempts at islet encapsulation have focused on development of the method, with relatively little emphasis on in vivo evaluation of the technology, and particularly on the maintenance of functional beta cells in an allogeneic environment. Teramura and Iwata demonstrated that coating of islet surfaces with PEG-lipid and urokinase could effectively protect against blood-mediated inflammatory reactions in a syngeneic mouse model of transplantation [23]. However, a recent study using conformal coating to encapsulate islets via layer-by-layer deposition of poly(L-lysine)-gPEG-(biotin) and streptavidin failed to show any improvement in the survival and function of the implanted allo-islets [19]. Effective immunoprotection using nano-coating thus remains a challenge in islet transplantation.

Layer-by-layer nanofilm deposition has been studied extensively in connection with the coating of biodevices such as implants in biomedical applications [29, 30]. In the current study, we aimed to adapt this technology to produce a conformal nano-coating for islets, which covers individual islets in nano-layers that possess anti-coagulation and antiinflammatory properties [31, 32], thus achieving effective encapsulation without significantly increasing the size of the islet. Charged linear polysaccharides carrying phosphorylcholine (PC) modification were used as coating materials (Fig. 1), with the binding being based on electrostatic interactions. As demonstrated previously [33], layer-by-layer deposition of alginate-chitosan nano-layers provides a mild process (near physiological conditions) that does not involve covalent cross-linking, it is therefore able to leave the cell surfaces and cell interior undisturbed. In this study, we investigated the localised immune protective effects of biopolymer nano-coating of islets and tested whether nanoscale encapsulation improves early outcomes from syngeneic and allogeneic islet transplantation in the commonly used streptozotocin-induced mouse model of type 1 diabetes.

\section{Methods}

A more detailed description of research design and methods, including regents and materials, is available in the electronic supplementary material (ESM Methods).

Experimental animals For syngeneic transplantation, male C57BL/6 mice aged 8 weeks and weighing 20-25 g (Charles River, Margate, UK) were used as donors and recipients of grafts. For allogeneic transplantation, male $\mathrm{C} 57 \mathrm{BL} / 6$ mice were used as islet graft recipients and Balb/ $\mathrm{c}$ mice as tissue donors. Recipient mice were made diabetic by a single i.p. streptozotocin injection $(180 \mathrm{mg} / \mathrm{kg}$; SigmaAldrich, Poole, UK) 5-6 days prior to transplantation and those with a non-fasting blood glucose concentration of 

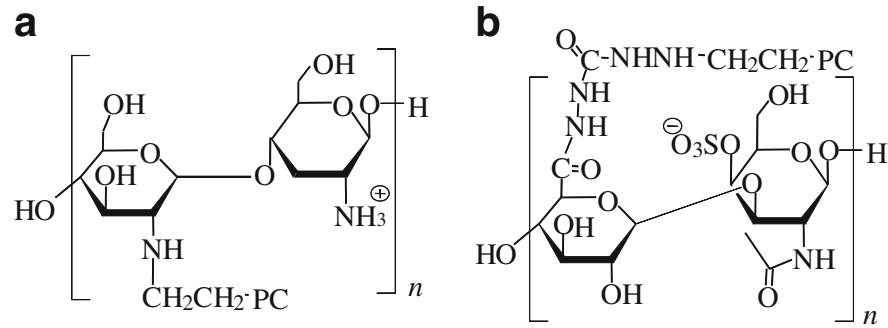

C<smiles>C[N+](C)=CCP(=O)([O-])OC#P</smiles>

Fig. 1 Chemical structure of the PC moiety-functionalised polysaccharides used in the nano-scale encapsulation. (a) PC-modified (40\% of the amine groups) chitosan (protonated); (b) PC-modified (100\% of the carboxylic group) chondroitin-4-sulphate (deprotonated); and (c) PC

$\geq 20 \mathrm{mmol} / \mathrm{l}$ were used as recipients. All animal procedures were approved by our institution's Ethics Committee and carried out under licence, in accordance with the UK Home Office Animals (Scientific Procedures) Act 1986.

Islet isolation Islets were isolated from mice by injecting collagenase (type XI, $1 \mathrm{mg} / \mathrm{ml}$; Sigma-Aldrich) into the pancreas via the common bile duct, followed by digestion for $10 \mathrm{~min}$ at $37^{\circ} \mathrm{C}$. The islets were purified by centrifugation (3,500 rpm, $25 \mathrm{~min}$; Universal 320R, Hettich Zentrifugen, Tuttingen, Germany) in a density gradient (Histopaque1077; Sigma-Aldrich). Before being used for encapsulation, the purified islets were incubated for $16 \mathrm{~h}$ in RPMI-1640 medium (Sigma-Aldrich) that was supplemented with $10 \%$ fetal bovine serum, $100 \mathrm{U} / \mathrm{ml}$ penicillin and $0.1 \mathrm{mg} / \mathrm{ml}$ streptomycin.

Nano-coating of islets The working solutions of the polysaccharides, including PC-chitosan, alginate and PC-modified chondroitin-4-sulphate (1 mg/ml; Fig. 1), were prepared by dissolving the solids in Hanks' buffered salt solution (HBSS; PAA Laboratories, Pasching, Austria), which was $\mathrm{pH}-$ adjusted to $6.9-7.0$ and supplemented by $2 \mathrm{mmol} / \mathrm{C} \mathrm{CaCl}_{2}$. Positively charged chitosan-PC and negatively charged alginate (unmodified) were alternately deposited into a multilayer film on individual islets in suspension in a $1.5 \mathrm{ml}$ Eppendorf tube. Cationic chitosan-PC $(400 \mu \mathrm{l})$ was added first to a suspension of the islets (pre-washed with HBSS) to form a seeding layer. After 5 min deposition time, two intermediate washings with the buffer were made using gravity sedimentation for 1 to $2 \mathrm{~min}$ to remove any excess, unadsorbed chitosan-PC. Subsequently, anionic alginate $(400 \mu \mathrm{l})$ was adsorbed in the same manner. This process was repeated $n$ times per batch of islets according to the following layering scheme: islets/(chitosan-PC-alginate layers) ${ }_{n}$, where $n$ represents the number of bilayers. Finally, a PC-modified chondroitin-4-sulphate $(400 \mu \mathrm{l})$ layer was added as the outermost layer in the same manner as for alginate. Some loss of islets was incurred, totalling $<10 \%$ for a typical eight-layer coating process. All encapsulation solutions were filtered through a sterile $0.2 \mu \mathrm{m}$ membrane filter cartridge. All coating, washing and sample solutions were kept on ice during the coating process.

Dynamic insulin secretion from the encapsulated islets $\mathrm{En}-$ capsulated islets were incubated for $16 \mathrm{~h}\left(37^{\circ} \mathrm{C}\right)$ before perifusion. The rate and patterns of in vitro insulin secretion from encapsulated and control islets were assessed using a temperature-controlled $\left(37^{\circ} \mathrm{C}\right)$ multi-channel perifusion system, as described previously [34]. Briefly, 40 islets were loaded on to nylon filters in Swinnex filter holders (Millipore, Cork, Ireland) and perifused with a bicarbonate-buffered physiological salt solution (Gey \& Gey buffer, made in house) supplemented by $2 \mathrm{mmol} / \mathrm{l} \mathrm{CaCl} 2,0.5 \mathrm{mg} / \mathrm{ml}$ bovine serum albumin and a concentration of glucose as indicated below. Fractions were collected every 2 min during (1) a $10 \mathrm{~min}$ perifusion period with buffer containing $2 \mathrm{mmol} / \mathrm{l}$ glucose, (2) a $20 \mathrm{~min}$ perifusion with $20 \mathrm{mmol} / \mathrm{l}$ glucose and (3) an additional $20 \mathrm{~min}$ perifusion with $2 \mathrm{mmol} / \mathrm{l}$ glucose. Insulin content was assessed by radioimmunoassay [34].

Transplantation of encapsulated islets in diabetic mice Mice were anaesthetised by inhalation of isoflurane and transplanted with 300 islets under the kidney capsule, according to a procedure reported previously [35]. Briefly, a lumbar incision was made, the kidney exposed and an incision made in the capsule. Encapsulated and control islets that had been centrifuged into pellets in PE50 polyethylene tubing (Becton Dickinson, Franklin Lakes, NJ, USA) were placed underneath the kidney capsule using a Hamilton syringe (Fisher, Two Rivers, WI, USA). All islets were transplanted with a delay of no more than $2 \mathrm{~h}$ after encapsulation.

In vivo function of islet graft The body weight and blood glucose concentrations of recipient mice were monitored every 1-2 days. Reversal of hyperglycaemia was defined as non-fasting blood glucose concentrations $\leq 11.1 \mathrm{mmol} / 1$ on at least two consecutive readings. In cured animals, we assessed the in vivo function of the transplanted islets by an intraperitoneal glucose tolerance test at 1 month after transplantation. Weight-matched, non-diabetic, non-transplanted male C57BL/6 mice were used as controls. Fasting blood 
glucose concentrations were measured prior to an i.p. injection of $2 \mathrm{~g} / \mathrm{kg}$ of glucose dissolved in saline solution and then after 15, 30, 60, 90 and $120 \mathrm{~min}$. The islet graft-bearing kidneys were removed 1 or 2 days later to assess whether graft removal would result in a reversion to hyperglycaemia. Other mice in which the graft was rejected in less than 28 days were killed and the graft-bearing kidney removed for histological analysis.

Immunohistochemistry Detailed descriptions of histological and immunohistological analysis of the graft-bearing kidneys is available in the ESM Methods.

Statistical analysis Independent $t$ tests were used to test for significant difference between individual groups of the cytokine assay results and glucose tolerance data. Values of $p<$ 0.05 were considered significant.

\section{Results}

Deposition of coating layers on islets We used a nano-scale encapsulation approach that uses spontaneous deposition of alternate layers of charged linear polysaccharides (PCchitosan/alginate) as coating materials, with a final layer of PC-modified chondroitin-4-sulphate, as illustrated in Fig. 2. The nano-coated C57BL/6 mouse islets showed better physical strength than the uncoated ones, as evidenced by an increase of $20-30 \%$ in packed tissue volume for the same amounts of islets when loaded in PE50 polyethylene transplantation tubing.

The deposition of eight layers was assessed by fluorescence microscopy following the incorporation of two layers of PC-chitosan tagged with FITC in the fifth and seventh layers. As shown in Fig. 3a, the addition of the FITC-tagged layer to the islets resulted in strong fluorescence localised on the surface of the islets, which is consistent with their extracellular architecture and indicates uniform coverage.

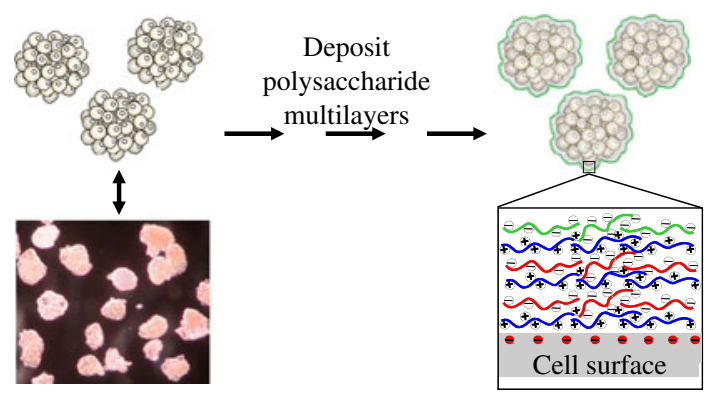

Fig. 2 Schematic representation of the nano-scale encapsulation of pancreatic islets, with layer-by-layer deposition of charged polysaccharide multilayers on individual islet surfaces. Blue lines with + symbol, chitosan-PC; red lines with - symbol, alginate; green lines with - symbol, chondroitin-4-sulphate-PC
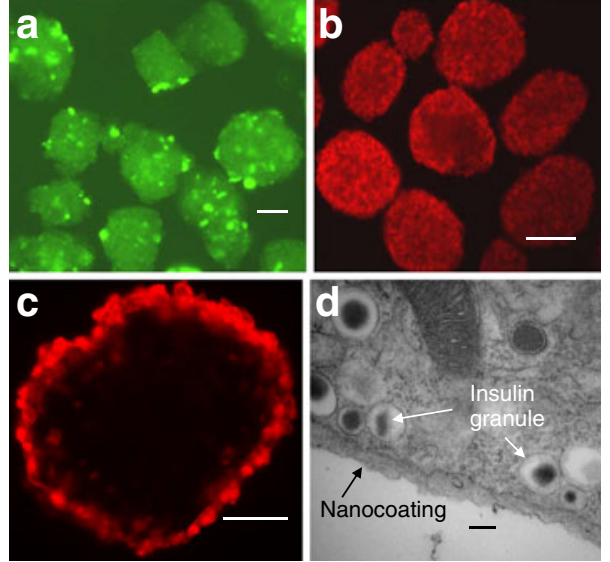

Fig. 3 Confirmation of the nano-scale encapsulation of mouse islets. a Deposition of eight layers with incorporation of two layers of PCchitosan tagged with FITC into the fifth and seventh layers. Scale bar $50 \mu \mathrm{m}$. b Fluorescence labelling of islets by incorporation of Alexa Fluor 647-tagged poly-L-lysine into the seventh layer as a fluorescent marker. Scale bar $100 \mu \mathrm{m}$. c Cross-section of a coated islet with an incorporated layer of Alexa Fluor 647-tagged poly-L-lysine (formalinfixed and paraffin-embedded), showing that the nano-layer appears to completely cover the islet surface. Scale bar $50 \mu \mathrm{m}$. d TEM micrograph of the cross-section of an islet encapsulated by an eight-layer coating (consisting of PC-chitosan, alginate and condroitin-4-sulphate as described in Methods, non-labelled). Islets were imaged shortly after completion of coating. Scale bar $100 \mathrm{~nm}$

A few bright spots found on the surfaces of islets may have been due to the higher surface charges that can occur on cell or extracellular surfaces. The above image (Fig. 3a) was taken shortly after encapsulation; however, the coating was found to be stable in culture throughout 10 days of monitoring (ESM Fig. 1). Due to the instability of FITC under paraffinembedding conditions, we also generated fluorescencelabelled islets by incorporating poly-L-lysine tagged with Alexa Fluor 647 in the seventh layer as a fluorescent marker (see ESM Methods) (Fig. 3b). The cross-section of the labelled islet showed that the exterior islet surfaces were completely covered and that the coating was localised extracellularly (Fig. 3c). Note that the thickness of the fluorescent layer ring could have been affected by the surface roughness of the islet and the position of the section taken from the islet.

The deposition of the non-labelled polysaccharide multilayers on the individual islets was further confirmed by high-resolution transmission electron microscopy (TEM) imaging of the encapsulated islets (Fig. 3d; ESM Methods). The ultrastructural image showed an intact coating consisting of nano-layers of approximately $80 \mathrm{~nm}$ thickness that covered the outer surface of cells on the islet periphery. The nano-layer was not found in the extracellular space between the islet cells. Insulin-secreting vesicles (dense-core granules) were seen by TEM to be aligned along the plasma membrane ready for exocytosis, suggesting that beta cells were healthy and unaffected by the coating. 
In vitro cytotoxicity exerted by the coating materials After maintenance in culture for up to 4 weeks, the survival of encapsulated mouse islet cells within eight layers was assessed using a two-colour fluorescence live-dead cell assay with the cell-permeable esterase substrate, fluorescein diacetate, and the cell-impermeant nucleic acid stain, propidium iodide (ESM Methods). Most of the cells in the islet were viable after 1 and 4 weeks of culturing, indicating that islets tolerated the multi-step nano-coating process with no or little detectable loss in cell viability (Fig. 4a, b).

In addition, no elevated level of apoptosis in the nanocoated islets was detected after $48 \mathrm{~h}$ in culture compared with non-encapsulated islets, as shown in Fig. 4c (see ESM Methods). The rate of islet cell apoptosis (determined as caspase-3/7 activity) in control islets was increased by approximately $620 \%$ following exposure to combined cytokines (IL-1 $\beta 1.7 \mathrm{ng} / \mathrm{ml}, \mathrm{TNF}-\alpha 1.7 \mathrm{ng} / \mathrm{ml}$ and IFN- $\gamma 3 \mathrm{ng} / \mathrm{ml}$ ), but this was significantly less in the nano-coated islets. The negative coating layer with alginate was more potent in inhibiting cytokine-induced cell damage than chondroitin-4-sulphatePC. Nano-coated islets were also protected against apoptosis mediated by complement (50\% rabbit serum; Fig. $4 \mathrm{~d})$. Chondroitin-4-sulphate-PC, an anti-coagulatory molecule $[31,32]$, was found to provide a better protective effect than alginate. Based on the findings above, the coating scheme adopted for our in vivo experiments used chitosan-PC and alginate in repeated layers, with the last layer being chondroitin-4-sulphate-PC.

Assessment of the in vitro function of nano-scale encapsulated islets Dynamic insulin secretion of the encapsulated islets was studied and compared with control using a perifusion system that simulates physiological conditions. As shown in Fig. 5, the four-layer encapsulated mouse islets retained glucose-induced insulin secretory responses. Increasing the glucose concentration from 2 to $20 \mathrm{mmol} / 1$ induced a rapid, biphasic and reversible stimulation of insulin secretion from islets encapsulated with four layers, with identical or similar response patterns from control islets. Increasing the nanofilm thickness to eight layers caused a small reduction in the kinetics and overall amount of insulin released, but the pattern and reversibility of the glucose-induced response were maintained.

In vivo assessment of nano-scale encapsulated islet function: syngeneic transplantation Figure 6a shows the data from islet transplantation under the kidney capsule from C57BL/6 donor mice (300 islets/graft) to diabetic recipients of the same strain. As expected, implantation of nonencapsulated islets as a control reversed hyperglycaemia and restored normoglycaemia for the duration of the study (4 weeks). Nano-scale encapsulated islets with four layers were equally effective in reversing hyperglycaemia, with a
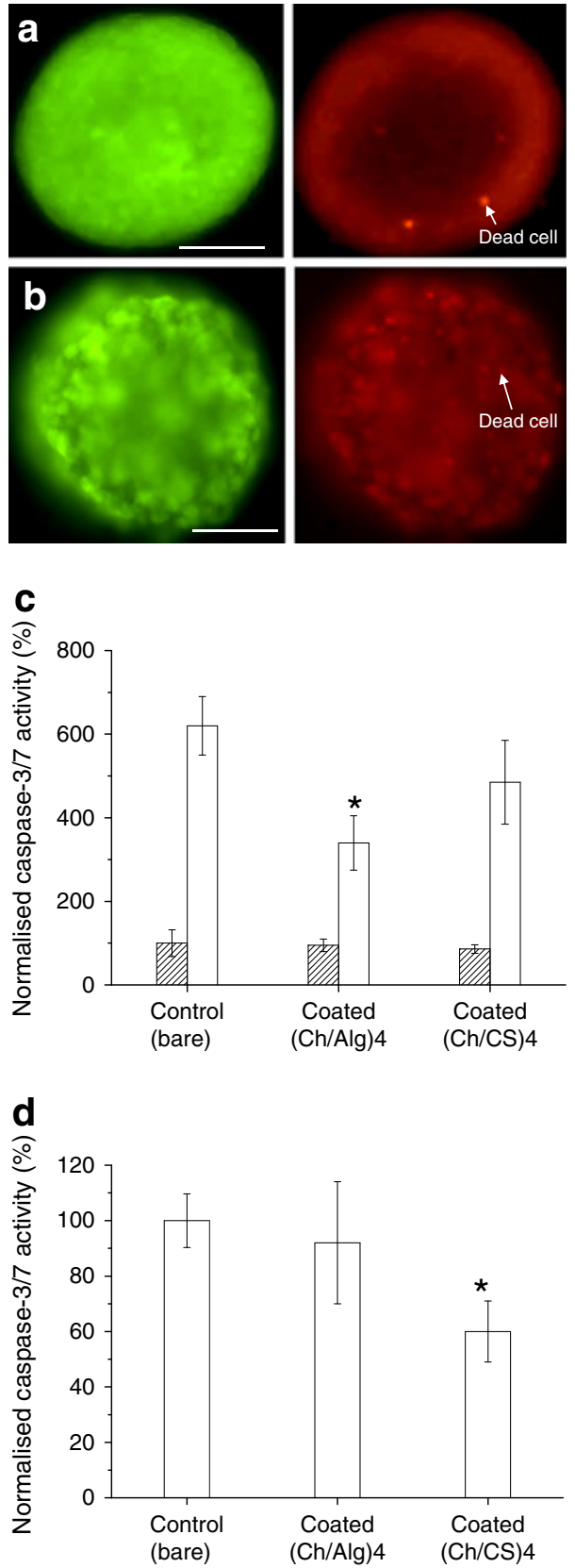

Fig. 4 Live-dead cell staining in coated islets. Nano-coated islets within eight layers of PC-chitosan and alginate were stained with fluorescein diacetate (green) and propidium iodide (red), and visualised (a) after 1 week and (b) at 4 weeks post-encapsulation of maintenance in culture. Green shows viable and red (dots) non-viable cells/islets. Similar data on uncoated islets can be found in our previous paper [28]. c Activity of caspase- 3 and -7 in the eight-layer-encapsulated and control (non-encapsulated) islets, with (white bars) and without (hatched bars) exposure to cytokine combination (IL- $1 \beta$, TNF- $\alpha$ and IFN- $\gamma$ ). Data were normalised against the value of control non-coated islets without cytokines. Ch, PC-chitosan; Alg, alginate; CS, chondroitin-4-sulphate. d Activity of caspase-3 and -7 in islets as above (c) after $24 \mathrm{~h}$ in culture with $50 \%$ rabbit serum. Data were normalised against the value of control non-coated islets exposed to the serum. Values $(\mathbf{c}, \mathbf{d})$ are mean $\pm \mathrm{SD} ; n=3 ;{ }^{*} p<0.05$ for PC-chitosan/ $\mathrm{Alg}$ or PC-chitosan/condroitin-4-sulphate-coated islets vs control islets 


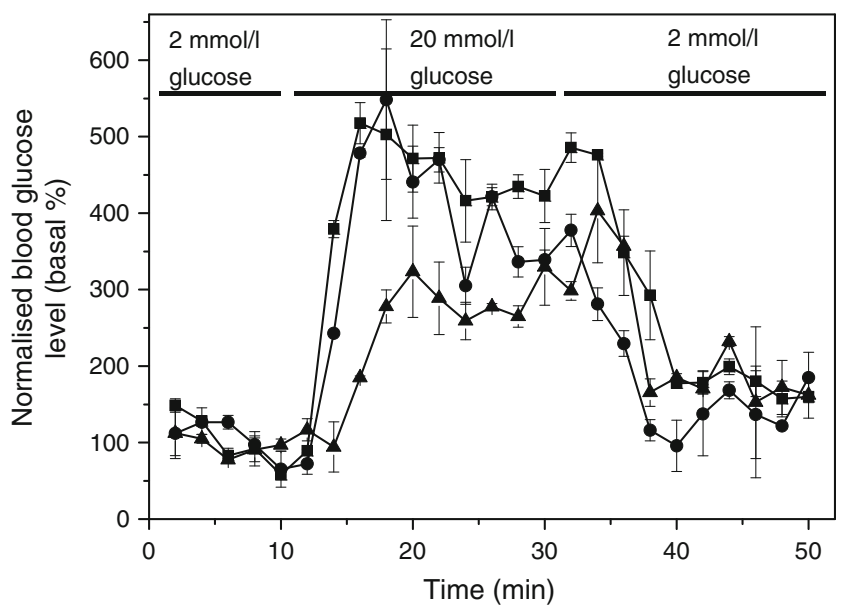

Fig. 5 Dynamic insulin production in vitro in control (non-encapsulated) and encapsulated (PC-chitosan, alginate, condroitin-4-sulphate) $\mathrm{Balb} / \mathrm{C}$ mouse islets in response to changes in extracellular glucose. Insulin secretion in response to the glucose challenge as a function of time was studied in a temperature-controlled cell perifusion system. Non-encapsulated islets (black squares) and encapsulated islets with four (black circles) and eight layers (black triangles) are shown. Periods of glucose stimulation and glucose concentration were as indicated. Values are mean $\pm \mathrm{SD} ; n=4$

time course similar to that of control islet grafts, in agreement with the functionality displayed in in vitro analyses. All mice reverted to the hyperglycaemic state on removal of the graft-bearing kidney (Fig. 6a). Subsequent histological assessment of graft material retrieved 28 days after implantation showed the presence of insulin-positive islet cell mass in the graft (Fig. 6b, c). There were no morphological differences between control and encapsulated islet grafts, with normal patterns of immunostaining for insulin, and no detectable major $\mathrm{T}$ cell (CD3 as the biomarker) and host macrophage (CD68 as the biomarker) infiltration at the graft sites (ESM Fig. 2).

In vivo assessment of nano-scale encapsulated islet function: allogeneic transplantation To assess whether encapsulation offers effective protection against immune rejection, we also performed studies using an allogeneic islet transplantation model, in which $300 \mathrm{Balb} / \mathrm{c}$ islets $(\mathrm{H}-2 \mathrm{~d})$ were transplanted below the kidney capsule of streptozotocin-induced diabetic C57BL/6 mice (H-2b). Transplanted islets reversed hyperglycaemia within 2-4 days. Animals implanted with control islets reverted to hyperglycaemia (blood glucose $>11.1 \mathrm{mmol} / \mathrm{l}$ ) within 10 to 14 days (median survival time 12 days) (Fig. 7a), consistent with graft rejection by the host immune system. Subsequent histological analysis of the nonencapsulated islet graft material revealed complete loss of the islet mass with little or no immunoreactive insulin being detectable (Fig. 7d). T lymphocytic and macrophage recruitment at the subcapsular implantation site was observed (ESM Fig. 3). Islets nano-encapsulated with four layers also reverted
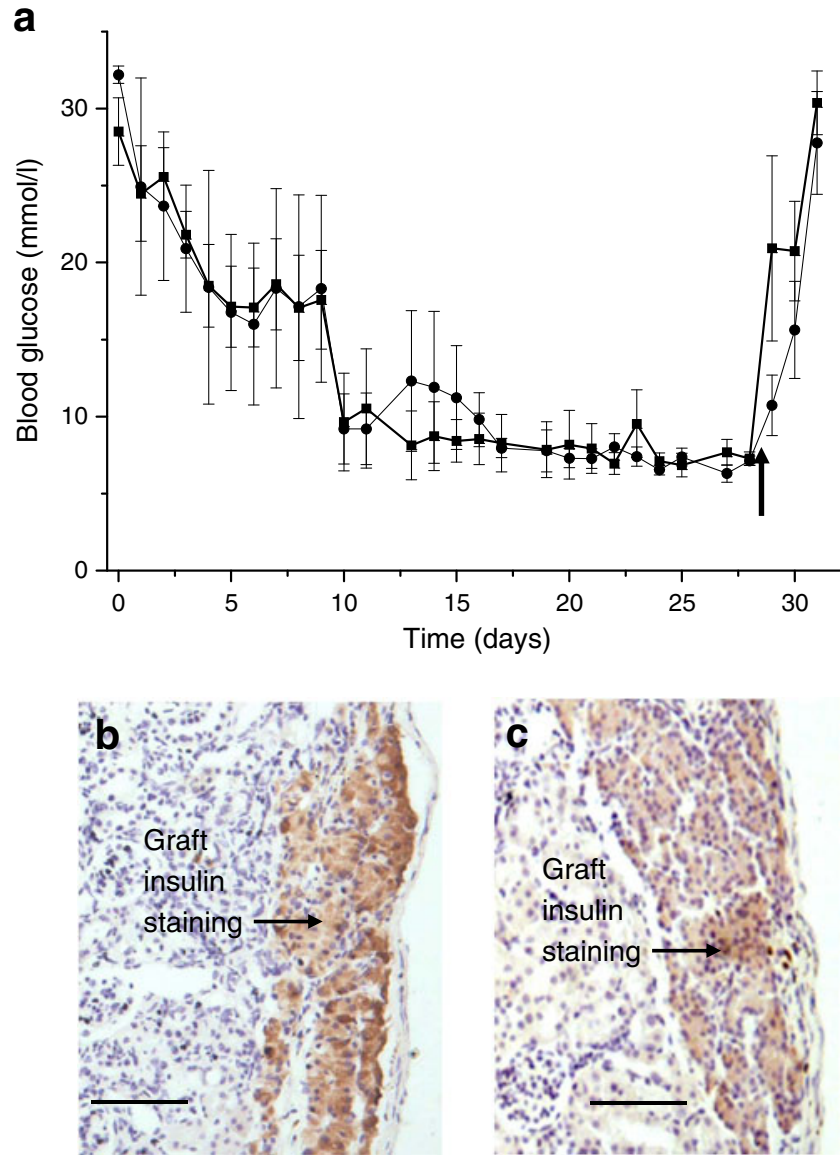

Fig. 6 Syngeneic transplantation of the encapsulated islets. a STZinduced hyperglycaemia was reversed by implantation of 300 islets of either control (black circles) islets or islets encapsulated with four layers (PC-chitosan, alginate, PC-chitosan, PC-condroitin-4-sulphate) (black squares). Graft removal (upright arrow) caused reversion to hyperglycaemia. Values are mean \pm SEM; $n=6$. b Histological analysis of recovered representative graft sections $(5 \mu \mathrm{m})$ of encapsulated and (c) non-encapsulated (control) islets using insulin immune-staining (brown). Scale bars $50 \mu \mathrm{m}$

to hyperglycaemia, albeit a few days later (median survival time 15 days), suggesting that this level of encapsulation was not sufficient to prevent immune rejection (ESM Fig. 4).

Protection from immune assault was more successful in a further study, in which we implanted islets coated with eight layers in the allogeneic model. In this study, the graft material was retrieved after about 1 month for histological analysis. Nano-scale encapsulated islets maintained normoglycaemia for 28-37 days (Fig. 7b) in five of seven mice. Reversion to hyperglycaemia for mice receiving encapsulated islet transplants was caused by graft-bearing kidney removal. Two mice receiving the encapsulated islets reverted to hyperglycaemia in less than 28 days due to rejection.

To determine whether transplanted islets secrete insulin and maintain glycaemic control in response to increases in blood glucose levels, we carried out intraperitoneal glucose tolerance tests at 4 weeks post-transplantation in mice that 
a

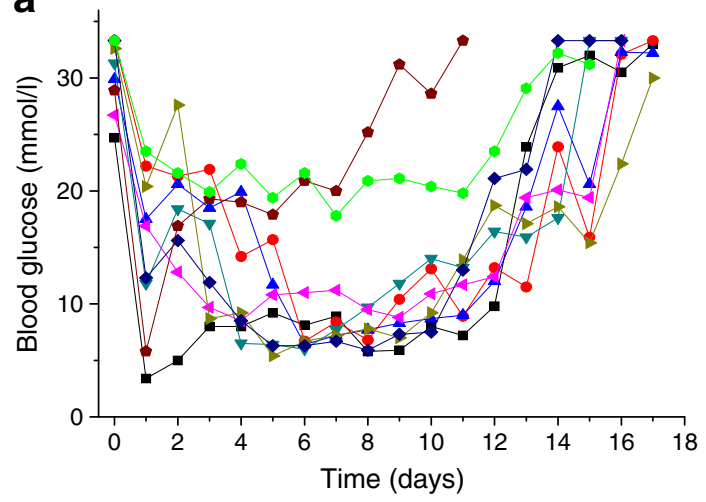

b

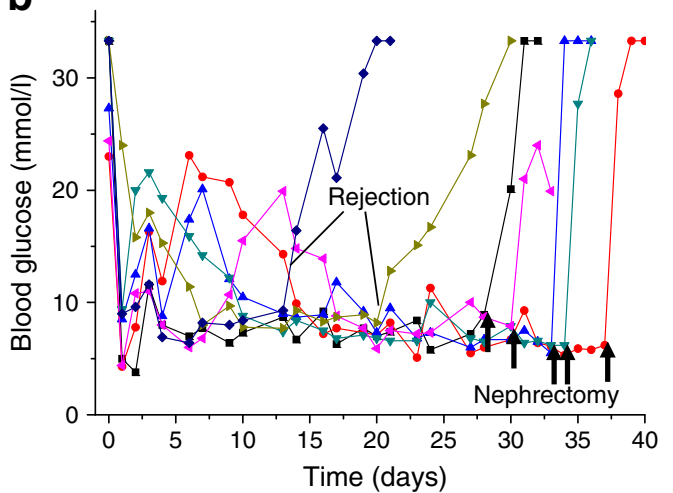

C

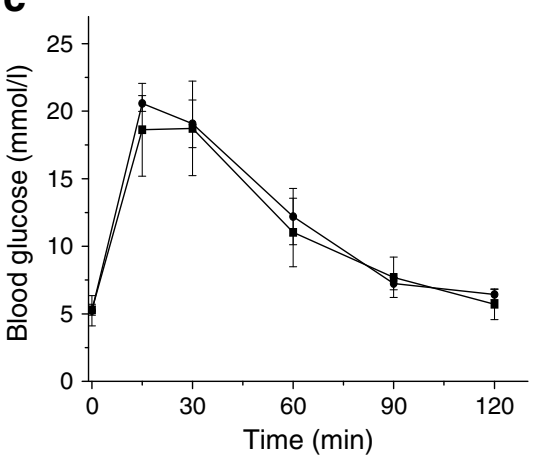

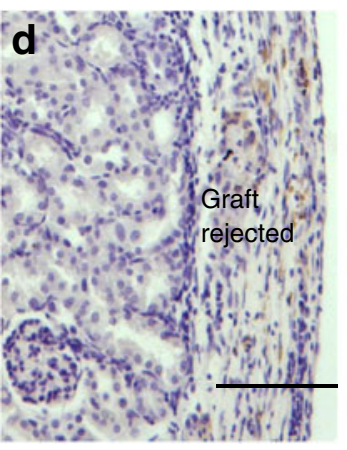

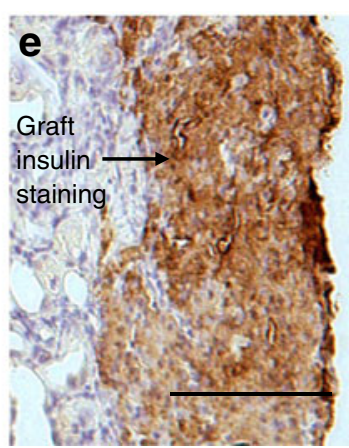

Fig. 7 Allogeneic transplantation of non-encapsulated (a) and eightlayer-encapsulated ((PC-chitosan and alginate $)_{3}+$ PC-chitosan + PCcondroitin-4-sulphate) (b) Balb/c islets reversed STZ-induced hyperglycaemia in diabetic C57BL/6 mice. Blood glucose concentrations of the individual animals (colour + symbol) are shown. Upright arrows (b), day of nephrectomy. c Intraperitoneal glucose tolerance tests were performed 4 weeks post-transplantation on mice with encapsulated

had remained normoglycaemic. Figure $7 \mathrm{c}$ shows that the change in glucose level was similar to that of non-diabetic mice. Histological analysis of the recovered grafts showed significant amounts of insulin-positive tissue (Fig. 7e) and no evidence of infiltration of $\mathrm{T}$ cells into the islets, although a few macrophages were detected (ESM Fig. 3).

\section{Discussion}

We describe here a new protocol for nano-scale encapsulation of pancreatic islet cells and for the first time demonstrate in an animal model improved post-transplant survival of such coated islet cells in comparison with uncoated islets.

Alternate layers of PC-grafted polysaccharides (chitosan and chondroitin-4-sulphate) were used with alginate as the coating materials, with the binding being based on electrostatic complexation. Multilayer film formation is possible because of charge reversal on the film surface after each adsorption step. The polysaccharides we used contain at least one charge in each monosaccharide unit, which serves as the binding site, offering hundreds of binding sites for islets (black circles) and on non-diabetic, non-transplanted mice as controls (black squares). Values (a-c) are mean \pm SEM; $n=5$; (c) $p>$ 0.05 for each data pair. d Histological analysis (insulin immunestaining, brown) of recovered representative graft-bearing kidney capsules in control non-encapsulated islets and (e) eight-layer-encapsulated islets. Scale bars $50 \mu \mathrm{m}$

each polysaccharide molecule, thus providing a strong affinity to the charged cellular surfaces. The rationale for using these polymers in the layer architecture was based on our in vitro studies, which identified potential complementand inflammation-inhibitory activities of these polysaccharides (Fig. 3c, d). Any success of islet encapsulation in extending graft survival in this study is likely to be dependent to a large part on the ability of the nano-coating to control the local transplant microenvironment and restrict immune cell infiltration, essentially blocking a variety of protein-protein interactions involved in complement (molecular mass $500 \mathrm{kDa}$ ), macrophage and $\mathrm{T}$ cell activation. In this regard, we introduced the protein-repelling zwitterionic PC modification (Fig. 1) in the coating constructs to minimise interactions between islets and the environment. The PC moiety, which is a component of plasma cell membranes, confers hydrophilicity, haemocompatibility and resistance to non-specific protein absorption, thus inhibiting the development of fibrosis, supporting endothelial cell growth [36] and also carrying anticoagulatory properties [37, 38], all of which should enhance islet survival in vivo and encourage host integration. In addition, the $40 \%$ amine group content of the PC modification for 
chitosan increased the solubility of the polysaccharide up to about $2 \mathrm{mg} / \mathrm{ml}$ under physiological $\mathrm{pH}$ conditions ( $\mathrm{pH} \sim 7.0)$.

Using the layer-by-layer deposition technique, a defined multilayer coating containing PC modification could be immobilised on the islet cell surfaces (ESM Fig. 5). The thickness $(80 \mathrm{~nm})$ of a typical eight-layer coating, as measured by TEM, is consistent with that previously reported with similar polymer sizes (200 nm with 20 layers) [39]. The addition of a polymer, chondroitin-4-sulphate, that carried a strongly ionic sulphate group into the complexation pair confers anti-coagulatory properties to the coating. It also contributes greatly to the stability of the layered nanofilm [40], making it a very durable coating even under harsh physiological conditions.

The nano-scale encapsulated mouse islets were found to preserve appropriate islet secretory function and survival in vitro. This indicates that the multilayers of the polysaccharides were deposited non-covalently on to the cell surfaces without perturbing cellular physiology or compromising cell survival. Pro-inflammatory cytokines, including IFN- $\gamma$, TNF- $\alpha$ and IL$1 \beta$, are major products of activated effective $\mathrm{T}$ cells and macrophages, and are known to be damaging to pancreatic islets via apoptosis induction [41]. In this study, we found that the nano-coating also rendered the islets less susceptible to cytokine- and complement-induced apoptosis (Fig. 4c, d). In addition, we found that the PC-displayed nano-coating effectively inhibited specific adsorption of large molecules of the immune systems (IgG, $150 \mathrm{kDa}$ ) on to the islet cell surfaces (ESM Fig. 6), indicating the effectiveness of the non-fouling PC-modification of the coating materials.

We used the syngeneic transplantation model to assess the in vivo secretory functionality of nano-scale encapsulated mouse islets and their ability to reduce hyperglycaemia and maintain normoglycaemia thereafter. These studies were used to avoid any influences of graft-versus-host immune rejection on islet function, while focusing on how the coating layers could impact on the immediate inflammatory reaction. Our in vivo tests using the syngeneic mouse transplantation model showed no deleterious responses from host animals to the coating materials, suggesting the materials used for encapsulation are non-toxic. Allo-transplantation studies in present work have shown that tailored encapsulating layers could optimise islet function post-implantation, allowing a degree of protection against inflammation and immune rejection in the majority of the studies. In the current study, nano-scale encapsulated islets were responsive to a hyperglycaemic environment, secreted appropriate amounts of insulin to restore normoglycaemia and survived for an extended period in vivo.

The failure of encapsulation to prevent rejection in two of the seven animals studied is of note and may indicate incomplete coating before transplantation or degradation at some time after transplantation. Future studies will need to test whether the robustness or completeness of the coating can be improved.
Since the purpose of our study was to prevent or reduce early-stage islet loss, we considered that a 4-5 week posttransplant observation period was appropriate. The rejection in controls normally occurs at 10-14 days (Fig. 7a), so the chosen study period showed that graft survival was twice as long. We have to look at grafts when the animals are normoglycaemic to determine whether there are any signs of graft destruction or whether the graft has a normal histological appearance. A long-term study of the function and graft survival with this multilayer coating scheme has not yet been performed. Nevertheless, unlike the arguably more expected durability of alginate microcapsules $[15,42]$, the nano-layers used in this study, while being non-biodegradable, are not expected or intended to last for many months under in vivo conditions. Rather, we propose that nano-scale encapsulation is an alternative technology that may promote engraftment by allowing intraportal administration and by limiting early islet loss caused by exposure to an inflammatory environment. In fact, our in vitro cytokine exposure study (Fig. 3c, d) confirmed that the encapsulation layers were protective against the cytotoxic effects of cytokines and complement proteins, as assessed by a reduction of apoptosis. Thus by the integration of graft tolerance induction mechanisms, the encapsulated environment may promote the development of regulatory $\mathrm{T}$ cells, which inhibit unintended host immune system activation, thereby achieving permanent survival of the transplanted islets [43, 44].

Studies are now needed to test whether modifications of the nano-layers will enhance engraftment and prevent rejection of islets in the longer term. This may involve: (1) incorporating bioactive molecules such as complement and coagulation inhibitors into the layers to prevent islet loss caused by the instant blood-mediated inflammatory reaction; (2) incorporating anti-inflammatory agents to reduce localised inflammation and fibrosis; or (3) incorporating natural and/or artificial extracellular matrices to provide optimal cell functioning after engraftment. Since there is little or no increase in size and volume of the islets after encapsulation, we expect this protocol will be potentially suitable for hepatic implantation via intraportal infusion. Such an option now needs to undergo experimental testing.

Funding The authors acknowledge the Engineering and Physical Sciences Research Council (EPSRC) (UK) Science and Innovation award (EP/D062861/1) for generous grant supports.

Contribution statement $\mathrm{ZlZ}$ contributed to the conception and design of the study, performed the experiments and wrote the manuscript. AK contributed to the interpretation of in vivo data. AJFK contributed to the conception and design of in vivo experiments. PMJ contributed to the conception and design of the study, and reviewed the manuscript. JCP contributed to the conception and design and reviewed/edited the manuscript. All authors have read and given critical input during preparation of the manuscript and all have approved the final version. 
Duality of interest The authors declare that there is no duality of interest associated with this manuscript.

\section{References}

1. Shapiro A, Lakey J, Ryan E et al (2000) Islet transplantation in seven patients with type 1 diabetes mellitus using a glucocorticoidfree immunosuppressive regimen. N Engl J Med 343:230-238

2. Biarnes M, Montolio M, Nacher V, Raurell M, Soler J, Montanya E (2002) Beta-cell death and mass in syngeneically transplanted islets exposed to short- and long-term hyperglycemia. Diabetes 51:66-72

3. Davalli AM, Scaglia L, Zangen DH, Hollister J, Bonner-Weir S, Weir GC (1996) Vulnerability of islets in the immediate posttransplantation period. Dynamic changes in structure and function. Diabetes 45:1161-1167

4. Miao G, Ostrowski RP, Mace J et al (2006) Dynamic production of hypoxia-inducible factor-1alpha in early transplanted islets. Am J Transplant 6:2636-2643

5. Tokodai K, Goto M, Inagaki A et al (2010) Attenuation of crosstalk between the complement and coagulation cascades by $\mathrm{C} 5 \mathrm{a}$ blockade improves early outcomes after intraportal islet transplantation. Transplantation 90:1358-1365

6. Chatenoud L (2008) Chemical immunosuppression in islet transplantation - friend or foe? N Engl J Med 358:1192-1193

7. Wilson JT, Chaikof EL (2008) Challenges and emerging technologies in the immunoisolation of cells and tissues. Adv Drug Deliv Rev 60:124-145

8. Lim F, Sun AM (1980) Microencapsulated islets as bioartificial endocrine pancreas. Science 210:908-910

9. Duvivier-Kali VF, Omer A, Parent RJ, O'Neil JJ, Weir GC (2001) Complete protection of islets against allorejection and autoimmunity by a simple barium-alginate membrane. Diabetes 50:16981705

10. Elliott RB, Escobar L, Tan PL, Muzina M, Zwain S, Buchanan C (2007) Live encapsulated porcine islets from a type 1 diabetic patient $9.5 \mathrm{yr}$ after xenotransplantation. Xenotransplantation 14:157-161

11. Tuch BE, Keogh GW, Williams LJ et al (2009) Safety and viability of microencapsulated human islets transplanted into diabetic humans. Diabetes Care 32:1887-1889

12. King A, Sandler S, Andersson A (2001) The effect of host factors and capsule composition on the cellular overgrowth on implanted alginate capsules. J Biomed Mater Res 57:374-383

13. Foster JL, Williams G, Williams LJ, Tuch BE (2007) Differentiation of transplanted microencapsulated fetal pancreatic cells. Transplantation 83:1440-1448

14. Bohman S, Andersson A, King A (2006) No differences in efficacy between noncultured and cultured islets in reducing hyperglycemia in a nonvascularized islet graft model. Diabetes Technol Ther 8:536-545

15. Schneider S, Feilen PJ, Brunnenmeier F et al (2005) Long-term graft function of adult rat and human islets encapsulated in novel alginate-based microcapsules after transplantation in immunocompetent diabetic mice. Diabetes 54:687-693

16. Bohman S, King AJ (2008) Islet alpha cell number is maintained in microencapsulated islet transplantation. Biochem Biophys Res Commun 377:729-733

17. Dufrane D, Steenberghe M, Goebbels RM, Saliez A, Guiot Y, Gianello P (2006) The influence of implantation site on the biocompatibility and survival of alginate encapsulated pig islets in rats. Biomaterials 27:3201-3208

18. Lee DY, Yang K, Lee S et al (2002) Optimization of monomethoxypolyethylene glycol grafting on the pancreatic islet capsules. J Biomed Mater Res 62:372-377
19. Wilson JT, Cui W, Chaikof EL (2008) Layer-by-layer assembly of a conformal nanothin PEG coating for intraportal islet transplantation. Nano Lett 8:1940-1948

20. Veerabadran NG, Goli PL, Stewart-Clark SS, Lvov YM, Mills DK (2007) Nanoencapsulation of stem cells within polyelectrolyte multilayer shells. Macromol Biosci 7:877-882

21. Miura S, Teramura Y, Iwata H (2006) Encapsulation of islets with ultra-thin polyion complex membrane through poly(ethylene glycol)phospholipids anchored to cell membrane. Biomaterials 27:58285835

22. Kizilel S, Scavone A, Liu X et al (2010) Encapsulation of pancreatic islets within nano-thin functional polyethylene glycol coatings for enhanced insulin secretion. Tissue Eng Part A 16:2217-2228

23. Teramura Y, Iwata H (2011) Improvement of graft survival by surface modification with poly(ethylene glycol)-lipid and urokinase in intraportal islet transplantation. Transplantation 91:271-278

24. Wilson JT, Krishnamurthy VR, Cui W, Qu Z, Chaikof EL (2009) Noncovalent cell surface engineering with cationic graft copolymers. J Am Chem Soc 131:18228-18229

25. Luan NM, Teramura Y, Iwata H (2011) Layer-by-layer coimmobilization of soluble complement receptor 1 and heparin on islets. Biomaterials 32:6487-6492

26. Teramura Y, Kaneda Y, Iwata H (2007) Islet-encapsulation in ultrathin layer-by-layer membranes of poly(vinyl alcohol) anchored to poly(ethylene glycol)-lipids in the cell membrane. Biomaterials 28:4818-4825

27. Tatsumi K, Ohashi K, Teramura $Y$ et al (2012) The non-invasive cell surface modification of hepatocytes with PEG-lipid derivatives. Biomaterials 33:821-828

28. Zhi ZL, Liu B, Jones PM, Pickup JC (2010) Polysaccharide multilayer nanoencapsulation of insulin-producing beta-cells grown as pseudoislets for potential cellular delivery of insulin. Biomacromolecules 11:610-616

29. Tang Z, Wang Y, Podsiadlo P (2006) Biomedical applications of layer-by-layer assembly: from biomimetics to tissue engineering. Adv Mater 18:3203-3224

30. Macdonald ML, Samuel RE, Shah NJ, Padera RF, Beben YM, Hammond PT (2011) Tissue integration of growth factor-eluting layer-by-layer polyelectrolyte multilayer coated implants. Biomaterials 32:1446-1453

31. Campo GM, Avenoso A, Campo S et al (2009) Glycosaminoglycans modulate inflammation and apoptosis in LPS-treated chondrocytes. J Cell Biochem 106:83-92

32. Senzolo M, Coppell J, Cholongitas E (2007) The effects of glycosaminoglycans on coagulation: a thromboelastographic study. Blood Coagul Fibrinolysis 18:227-236

33. Mansouri S, Merhi Y, Winnik FM, Tabrizian M (2011) Investigation of layer-by-layer assembly of polyelectrolytes on fully functional human red blood cells in suspension for attenuated immune response. Biomacromolecules 12:585-592

34. Hauge-Evans AC, King AJ, Carmignac D et al (2009) Somatostatin secreted by islet delta-cells fulfills multiple roles as a paracrine regulator of islet function. Diabetes 58:403-411

35. Rackham CL, Chagastelles PC, Nardi NB, Hauge-Evans AC, Jones PM, King AJ (2011) Co-transplantation of mesenchymal stem cells maintains islet organisation and morphology in mice. Diabetologia 54:1127-1135

36. Tardif K, Cloutier I, Miao Z et al (2011) A phosphorylcholinemodified chitosan polymer as an endothelial progenitor cell supporting matrix. Biomaterials 32:5046-5055

37. Monge S, Canniccioni B, Graillot A, Robin JJ (2011) Phosphoruscontaining polymers: a great opportunity for the biomedical field. Biomacromoleules 12:1973-1982

38. Habara S, Mitsudo K, Kadota K et al (2011) Serial clinical and angiographic follow-up after phosphorylcholine-coated stent implantation. Intern Heart J 52:88-91 
39. Dubas ST, Schlenoff JB (2001) Polyelectrolyte multilayers containing a weak polyacid: construction and deconstruction. Macromolecules 34:3736-3740

40. Hoogeveen NG, Stuart MAC, Fleer GJ (1996) Formation and stability of multilayers of polyelectrolytes. Langmuir 12:36753681

41. Cetkovic-Cvrlje M, Eizirik DL (1994) TNF-alpha and IFN-gamma potentiate the deleterious effects of IL-1 beta on mouse pancreatic islets mainly via generation of nitric oxide. Cytokine 6:399-406
42. Soon-Shiong P, Feldman E, Nelson R et al (1993) Long-term reversal of diabetes by the injection of immunoprotected islets. Proc Natl Acad Sci USA 90:5843-5847

43. Lewis EC, Mizrahi M, Toledano M et al (2008) alpha1-Antitrypsin monotherapy induces immune tolerance during islet allograft transplantation in mice. Proc Natl Acad Sci USA 21:16236-16241

44. Gibly RF, Graham JG, Luo X, Lowe WL Jr, Hering BL, Shea LD (2011) Advancing islet transplantation: from engraftment to the immune response. Diabetologia 54:2494-2505 